\title{
In search of quorum effects in metacommunity structure: Species co-occurrence analyses
}

David G. Jenkins

University of Central Florida

Find similar works at: https://stars.library.ucf.edu/facultybib2000 University of Central Florida Libraries http://library.ucf.edu

This Article is brought to you for free and open access by the Faculty Bibliography at STARS. It has been accepted for inclusion in Faculty Bibliography 2000s by an authorized administrator of STARS. For more information, please contactSTARS@ucf.edu.

\section{Recommended Citation}

Jenkins, David G., "In search of quorum effects in metacommunity structure: Species co-occurrence analyses" (2006). Faculty Bibliography 2000s. 6261.

https://stars.library.ucf.edu/facultybib2000/6261

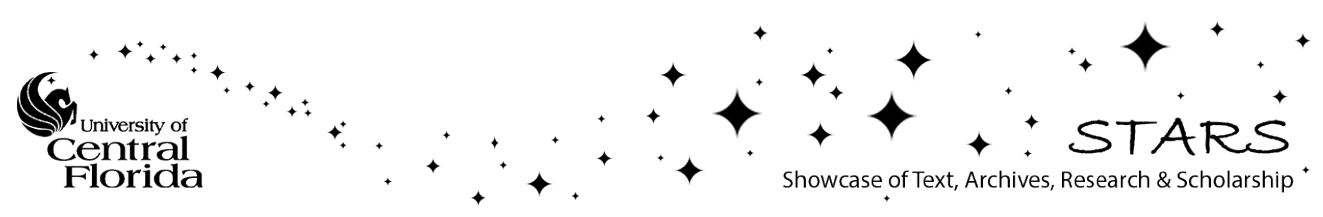




\title{
IN SEARCH OF QUORUM EFFECTS IN METACOMMUNITY STRUCTURE: SPECIES CO-OCCURRENCE ANALYSES
}

\author{
DAVID G. JENKINS ${ }^{1}$ \\ Department of Biology, University of Central Florida, 4000 Central Florida Boulevard, Orlando, Florida 32816-2368 USA
}

\begin{abstract}
Alternative models of community assembly emphasize regional, stochastic, dispersal-based processes or local, deterministic, niche-based processes. Community ecology's historical focus on local processes implicitly assumes that local processes surpass regional processes over time or across space to derive nonrandom metacommunity structure (i.e., a quorum effect). Quorum effects are expected late in succession among nearby sites, whereas quorum effects are not expected early in succession among distant sites. I conducted a metaanalysis of zooplankton data sets encompassing time scales of one to thousands of years and spatial scales of $<1 \mathrm{~m}$ to thousands of kilometers. Species co-occurrence analyses statistically evaluated presence/absence patterns relative to random patterns obtained with Monte Carlo null models. A series of weighted analyses was conducted and alternative randomization algorithms and null models were evaluated.

Most zooplankton metacommunities were randomly structured in unweighted analyses, and the distribution of significant structure did not follow quorum effect predictions. Weighted analyses (e.g., by habitat area) revealed significant, nonrandom structure in most zooplankton metacommunities, but the distribution of significant structure still did not adhere well to quorum effect predictions. Finally, additional weighting for study scale (number of sites) nullified most significant area-weighted structure, and again, the distribution of significant structure did not follow quorum effect predictions. Overall, a quorum effect was not supported, perhaps related to zooplankton life histories and energetics and/or the quorum effect itself. Results at the presence/absence level of resolution indicated that local processes did not generally override regional processes over time or across space to drive community structure. A full integration of dispersal- and niche-based concepts in metacommunity dynamics will be most fruitful for unraveling community assembly. Species co-occurrence analyses were scale dependent (habitat area and study size). Future analyses should use weights for important factors (e.g., habitat area), and meta-analyses should include study scale as an additional factor contributing to apparent patterns.
\end{abstract}

Key words: community assembly; local scale; meta-analysis; regional scale; succession; zooplankton.

\section{INTRODUCTION}

Local biotic and abiotic regulation of community composition has been central to community ecology and bears a long history of descriptive and experimental studies (McIntosh 1985, Oksanen 1991, Morin 1999). Ecologists often draw a dichotomy between deterministic, niche- based community processes and neutral approaches that emphasize stochastic, regional, dispersal-based processes (e.g., Clements [1916] vs. Gleason [1926]; Hubbell [2001] vs. Chase and Leibold [2003]). Dichotomies help advance ecology via clear, testable hypotheses but do not fully address intermediate complexities and contingencies typical of nature. In fact, the emerging discipline of metacommunity ecology (Leibold et al. 2004, Chase 2005) is based on the above works and others (e.g., Ricklefs 1987, 2004, Robinson and Dickerson 1987, McCormick et al. 1991, Cornell

Manuscript received 16 August 2005; revised 9 December 2005; accepted 13 December 2005. Corresponding Editor: M. Holyoak.

${ }^{1}$ E-mail: dgjenkin@mail.ucf.edu and Lawton 1992, Belyea and Lancaster 1999, Cornell 1999, Shurin 2000, Chave 2004, Fukami 2004, Kneitel and Chase 2004, Price and Morin 2004) that recognize multiple dispersal- and niche-based processes.

Ecology's historical focus on local dynamics has implicitly presumed that systems are older than their community assembly time (Mouquet et al. 2003) and have reached a "quorum" (Jenkins and Buikema 1998) whereby local regulators of community composition dominate over regional processes. A quorum occurs when the effect of all local biotic processes (e.g., competition and predation) plus abiotic tolerance limits exceeds the effects of regional dispersal processes on community composition. Highly connected communities with frequent dispersal (e.g., Michels et al. 2001, Cottenie et al. 2003) or those composed of rapid colonizers may reach a quorum rapidly, while slow arrival rates delay a quorum. The quorum metaphor is based on the toofamiliar experience of a committee meeting, which can attain quorum before all members are present. Likewise, a transition from regional to local regulation of community composition could occur before a commun- 
TABLE 1. Conceptual interaction of temporal and spatial quorum effects.

\begin{tabular}{ccc}
\hline \hline & \multicolumn{2}{c}{ Spatial quorum effect } \\
\cline { 2 - 3 } Temporal quorum effect & $\begin{array}{c}\text { Local } \\
\text { (quorum) }\end{array}$ & $\begin{array}{c}\text { Regional } \\
\text { (non-quorum) }\end{array}$ \\
\hline
\end{tabular}

Early (pre-quorum) contingent on system non-quorum conditions

Late (post-quorum) quorum conditions contingent on system

Notes: Categories are acknowledged to be simplistic representations of spectra. Results in early/local and late/regional systems are predicted to be contingent on the system (isolation, area, heterogeneity, etc.), species vagility, and study design (e.g., timing, scale, etc.). Temporal and spatial scales are predicted to determine whether a quorum effect occurs; see Introduction for further explanation.

ity is saturated or reaches an immigration-extinction equilibrium. The concept of a quorum effect attempts to address the general question "Do local or regional processes regulate community composition?" Should a general quorum effect be detected, it is then logical to test more specific mechanisms (Platt 1964). For example, competitive priority effects (Paine 1977) are a locally acting residual of colonization history (Jenkins and Buikema 1998) that can be lumped with other local, biotic interactions if one is first asking "Do local or regional processes regulate community composition?" If a quorum effect was demonstrated, then priority effects could be evaluated relative to other, local biotic processes to tease apart the nuances of a quorum effect.

The quorum metaphor can also be applied to spatial patterns: a quorum is more likely in systems within shorter distances. For example, Shurin et al. (2000:3070) analyzed zooplankton local:regional species richness patterns (Cornell and Karlson 1996) and concluded that "dispersal limitation only plays a detectable role at very large scales, and that local interactions dominate in generating differences in composition among lakes separated over shorter distances." However, the use of local:regional species richness relationships has since been deemed "unsuitable" (Hillebrand and Blenckner 2002) and "unwarranted" (He et al. 2005) for indicating the importance of local interactions (Hillebrand 2005). Because metacommunity ecology focuses on spatial patterns and linkages among communities (Kneitel and Chase 2004, Leibold et al. 2004, Chase 2005), alternative analytical approaches in the consideration of spatial dynamics may be helpful.

Of course, ecosystems operate in both time and space, and it would be most realistic and beneficial to simultaneously consider temporal and spatial quorum effects and their interactions (Table 1). For example, an ecosystem that is late in succession and near other, similar ecosystems (late/local systems in Table 1) may be expected to be have reached quorum (i.e., community composition is governed by local processes because most influential species have had an opportunity to colonize).
In contrast, early/regional ecosystems may not have reached quorum because not enough time has elapsed for influential species to colonize over these greater distances (Table 1). Other combinations of temporal and spatial conditions lead to conflicting predicted outcomes (Table 1) and so detection of quorum effects in those cases is likely contingent upon multiple factors (e.g., variance in habitat area or conditions among sites, species vagility, and study design) and cannot be predicted easily in advance.

Species co-occurrence analyses provide an approach to test for quorum effects in data sets that differ in time and space. Species co-occurrence analyses originated with Diamond's (1975) analysis of checkerboard patterns among island avifauna and sparked debate in community ecology (e.g., Connor and Simberloff 1979, 1983, Gilpin and Diamond 1984). Since then, statistical problems have been resolved (Gotelli and Graves 1996, Gotelli and Entsminger 2003), and species co-occurrence analyses are increasingly applied to evaluate community composition in diverse systems (e.g., Gotelli and McCabe 2002, Kobza et al. 2004, Peres-Neto 2004, Badano et al. 2005, Heino and Soininen 2005).

I analyzed zooplankton species presence/absence data from a variety of sources to test the hypothesis that significant species co-occurrence patterns are consistent with quorum effect predictions in Table 1. This hypothesis (and application of species co-occurrence analyses) is more general than that regarding the formation of checkerboard patterns by competition (Diamond 1975) because predation, local abiotic conditions, and/or competitive exclusion may contribute to quorum effects. Similar use of distributional data sets to indicate competitive exclusion has been debated at length (Gotelli and Graves 1996), but competitive guilds alone were not analyzed here: zooplankton communities also include predators (e.g., cyclopoid copepods and Chaoborus). Instead, I attempted to detect the combined effects of all local biotic and abiotic interactions that may structure zooplankton communities, which is a test of the quorum effect and a logical prerequisite to analyses of more specific mechanisms (e.g., competition).

Why use presence/absence data when experiments are more likely to detect subtle quorum effects on density (e.g., Shurin 2000)? First, an intensive search for subtle quorum effects (e.g., density, biomass) is better justified if more obvious effects (e.g., species presence/absence) are first found to exist (Platt 1964). Second, comparable experimental data are not available among diverse localities and regions to permit a more detailed test of generality for these concepts. Third, species co-occurrence analyses (Gotelli and Graves 1996, Gotelli and Entsminger 2003) can account for different levels of sampling effort among studies and site differences (as weights) that may not be addressed in experiments using natural ecosystems. Finally, even if those data were available, different experimental designs and scales may confound 
comparisons without means to compensate within analyses, as are afforded by species co-occurrence analyses.

Why focus on zooplankton communities? First, research related to temporal (Jenkins and Buikema 1998) and spatial (Shurin et al. 2000) quorum hypotheses dealt with these communities; it seems appropriate to first test these concepts with zooplankton at diverse scales and locations before extension to other communities. Second, a strong foundation of past research (Hutchinson 1967, Kerfoot and Sih 1987, Rothhaupt 1990) on local biotic and abiotic factors affecting zooplankton community structure indicates that quorum effects should occur in zooplankton communities. Conversely, this history potentially contrasts with random species co-occurrence patterns observed for invertebrates (other than eusocial ants) and other poikilotherms (Gotelli and McCabe 2002) in that abiotic and biotic local regulation of zooplankton community structure may not translate into significant quorum effects at the presence/absence level of detection. Therefore, an historically based expectation of quorum effects in zooplankton begs for closer examination. Third, much of our knowledge on zooplankton communities is based on experimentation, and a greater integration of biogeographic analyses (e.g., species cooccurrence analyses) with experimental results should be fruitful for ecology. Finally, ponds and lakes provide discrete habitats embedded in a terrestrial landscape, which enhances the ability to evaluate questions of distance and area relative to some intergrading terrestrial communities.

\section{Methods}

Eleven published and four unpublished data sets were assigned to each of four temporal/spatial classes (Table 1) based on available information (summarized in Appendix A). Subsets of five regional-scale data sets were analyzed to provide local-scale analyses at matching temporal scale. For example, tundra lakes at the northern extreme of the Swadling et al. (2000) largescale transect were used as a local subset. In total, 20 data sets (five for each of the four classes in Table 1; see Appendix A) were analyzed and hereafter are dubbed "systems." Analyzed systems ranged widely in spatial scale ( $2 \mathrm{~m}$ to $3976 \mathrm{~km}$ ), geographic location (used as a proxy for post-glacial age), number of species (11-142) and number of sample sites (4-127; Appendix A). Unpublished data sets were: Savannah River Site wetlands (South Carolina, USA; see Mahoney et al. 1990); Missouri River scour ponds (Missouri, USA; see Havel et al. 2000); Bluff Springs Sand Ponds (Illinois, USA; see Jenkins et al. 2003); and Discovery Bay rock pools (Jamaica; see Kolasa et al. 1996, Therriault and Kolasa 1999, Romanuk and Kolasa 2002).

Systems were assigned a priori to classes based on information contained in papers or provided by authors. For example, systems in Jamaican coastal rock pools and lakes across much of the northeastern United States were both assigned to the late/regional class because both systems exhibit strong habitat heterogeneity regardless of linear distance between sites, and both systems have likely existed for thousands of years (Appendix A). Because the a priori assignments of systems to temporal/ spatial classes may contribute to revealed patterns and conclusions, the effects of potential reassignments were also considered post hoc to evaluate the importance of class assignments on outcomes.

Each system's binary presence/absence data were analyzed for species co-occurrence pattern with EcoSim 7.0 (Gotelli and Entsminger 2002), using the mean number of "checkerboard units" ( $C$ score; Stone and Roberts 1990). The $C$ score provides a measure of species segregation without requiring that perfect checkerboard distributions exist (Gotelli 2000) and is superior to other indices in Type I and II error rates (Gotelli and Entsminger 2002). All simulation analyses were conducted with 5000 random iterations per data set (Gotelli 2000), and calculated $C$ scores were compared to distributions of random results. In a community structured by negative local interactions (e.g., competition, predation, abiotic limits) and thus in quorum conditions, the $C$ score should be significantly ( $P \leq$ $0.05)$ larger than expected by chance. Note that this application of species co-occurrence analysis extends its use beyond the traditional focus on competitive interactions to include all processes that may constrain community composition.

The details of species co-occurrence analyses are important, including the selection of appropriate null models (Gotelli and Graves 1996) and randomization algorithms (Gotelli and Entsminger 2003). The most appropriate null model per sampling design of each study was selected: the SIM2 model was used for singlesampling event ("sample list") data sets and the SIM9 model was used for multiple-sampling event ("island lists") data sets (Gotelli 2000). The sequential-swap randomization algorithm was used because it has low Type I and II error rates (Gotelli 2000, Gotelli and Entsminger 2002, 2003).

The above null model analyses treat sites equally. An important alternative is to weight sites for factors that may contribute to inter-site differences in community composition (e.g., habitat area; Fukami 2004). Analyses were weighted by habitat area and other factors described by the study authors as important to zooplankton community structure (e.g., hydroperiod; Appendix B). The relative size of a weight factor (e.g., area) adjusts the probability of a species occurring in a site during randomization, as opposed to an unweighted analysis that treats sites with equal probabilities. Multiple weighting factors were used in separate analyses if authors considered more than one factor to be important to zooplankton community structure. If a factor was important to community structure patterns, weighting with that factor was hypothesized to significantly alter calculated statistics. For example, if a 
species-area effect was actually responsible for unweighted species co-occurrence patterns, a significantly nonrandom pattern would become insignificant after weighting by area. Likewise, if the species-area effect confounded unweighted patterns, a nonsignificant pattern may become significant after weighting for habitat area. Weighting accounts for factors that may either mask or falsely indicate quorum effects, so I expected this approach to make the greatest difference for outcomes in data sets of early/local and late/regional classes that were expected to have results contingent on system conditions (Table 1).

Habitat area was the most common and dominant weighting factor among analyzed systems (Appendix B), but studies also varied in the number of sites (Appendix A). Area-weighted standard effect sizes were regressed against the number of sites, and then residuals were analyzed as "standard effect sizes" that have been weighted for both habitat area and study scale. Such a two-tiered weighting process is not part of EcoSim, but 95\% confidence intervals for standardized effect size are approximately -1.96 to 1.96 , as for $Z$ statistics, so that significance of residuals could be estimated from a $Z$ distribution. The same reasoning was applied to positive and negative residual values as was used in habitat-area weights: a negative value indicated that the study scale had falsely contributed to apparent checkerboard structure, while a positive value indicated that study scale had confounded weighted analyses and that checkerboard structure was better revealed after calculating study scale residuals.

The standardized effect size ([C score - mean simulated $C$ score]/standard deviation of simulated $C$ scores) was recorded to compare analyses (Gurevitch et al. 1992, Gotelli and Entsminger 2002). The effect of weighting factors on analyses was assessed as [weighted standard effect size] - [unweighted standard effect size]. Effects of time, space, sampling design (i.e., "sample list" vs. "island list" studies; Gotelli 2000), number of sites, and number of species on $C$ scores and standardized effect sizes were examined graphically and by regression.

The effects of time, space, and time-space interactions (Table 1) on standard effect sizes were also tested by factorial ANOVA, which enables testing for interaction effects (unlike nonparametric methods) and is often considered robust to assumption violations. However, ANOVA results should be interpreted cautiously and are not emphasized because of the following considerations. (1) Some local subsets were constructed from within some regional data sets to enable local-scale analyses at comparable temporal scales. This approach was important to maintain consistent temporal scaling across spatial classes, but violated the assumption of independence among temporal/spatial classes. This also excluded nonparametric tests for independent samples (i.e., Kruskal-Wallis tests). (2) Standardized effect sizes of temporal classes were not normally distributed, and all treatments were heteroscedastic, despite transformations.

Linear regressions were computed between important study variables (number of sites, number of species) and output variables ( $C$ scores, standardized effect sizes) to evaluate the influence of study scale on computed results. Residuals from the standard effect size-number of sites regression were used as a second weighting variable to account for the effects of study scale on species co-occurrence analyses that were already weighted for habitat area. Statistics were computed with SPSS version 11.5 (SPSS, Chicago, Illinois, USA).

\section{RESUlts}

Results of analyses differed substantially from predictions and depended importantly on the use of weights for important factors (e.g., area and study scale). Unweighted analyses did not support a priori predictions. Results in early/local systems were predicted to be contingent on system conditions, and one of the five data sets exhibited checkerboard pattern significantly different from random (Table 2 and Appendix B). All early/ regional systems were predicted to lack significant checkerboard structure, but three of five systems were significantly more structured than randomly assembled communities. All late/local systems were predicted to exhibit significant checkerboard structure (i.e., they have reached quorum), but all systems were not significantly different from random. Finally, quorum conditions were expected to be contingent on the system in late/regional data sets, and three of five showed a checkerboard pattern significantly different from random. Analysis of variance failed to detect significant effects of time $(P=$ $0.531)$, space $(P=0.908)$, or time-space interaction $(P=$ 0.676) on standard effect sizes, though these results must be considered cautiously given violations of assumptions. Overall, seven of 20 data sets had significant checkerboard structure, but patterns across the four temporal/ spatial classes did not fit predicted patterns well.

Weighted analyses detected significant species cooccurrence patterns more frequently (15 of 20 systems), indicating that quorum effects were more general than observed with unweighted analyses. Habitat area was significant in 13 of 17 systems for which area data differed among sites and were available (Table 2, Appendix B). In 12 of those 13 significant cases, areaweighted analyses yielded a positive change in standard effect size: area had significantly confounded unweighted analyses (through species-area effects) and weighting for habitat area typically helped reveal checkerboard patterns in species co-occurrence. Other weighting variables occasionally (four of 10 cases) yielded significant species co-occurrence patterns and were sparsely distributed among systems.

Weighting yielded negative effects in several systems: for example, system 2A (see Appendices A and B) was weighted by latitude in an attempt to represent the effect of degree-days on species richness (as stated by the study 
TABLE 2. Summary of species co-occurrence analyses (see Appendix B for details).

\begin{tabular}{lccr}
\hline \hline & \multicolumn{2}{c}{ Spatial quorum effect } \\
\cline { 2 - 3 } Temporal quorum effect & Local (quorum) & Regional (non-quorum) & Spatial difference \\
\hline Early (pre-quorum) & early/local (contingent) & early/regional (random) & 0.0 \\
Predicted & $0.50 \dagger$ & $0.60(5)$ & 0.40 \\
Unweighted & $0.20(5)$ & $0.50(4)$ & -0.25 \\
Area & $0.75(4)$ & $0.50(4)$ & 0.50 \\
Area + scale & $0.00(4)$ & late/regional (contingent) & $0.50 \dagger$ \\
Late (post-quorum) & late/local (quorum) & $0.60(5)$ & 0.60 \\
Predicted & 1.00 & $1.00(5)$ & -0.25 \\
Unweighted & $0.00(5)$ & $0.20(5)$ & \\
Area & $0.75(4)$ & 0.00 & 0.50 \\
Area + scale & $0.25(4)$ & -0.30 & \\
Temporal difference & & 0.20 & \\
Unweighted & 0.00 & 0.25 & \\
Area & Area + scale & & \\
\hline
\end{tabular}

Notes: Temporal/spatial classes are arranged as in Table 1, and results are summarized for analyses that were unweighted, areaweighted, and weighted for area + scale in each temporal/spatial class. Values are the proportion of species co-occurrence analyses either predicted or observed to be significantly nonrandom (i.e., indicated quorum effects; $P<0.05$ ). Parenthetical values are the number of analyses per temporal/spatial class; note that area data were not available for some studies. Temporal and spatial difference values (bottom row and right column, respectively) are the difference between scales (e.g., unweighted early/regional proportion - unweighted early/local proportion) as a measure of the effect of increased scale on analytical outcomes. Temporal and spatial scales are predicted to determine whether a quorum effect occurs; see Introduction for further explanation.

$\dagger$ A value of 0.50 (as in an equiprobable coin toss) is predicted because quorum vs. non-quorum conditions are contingent on multiple other factors and cannot be predicted in the absence of information on those conditions.

authors Hebert and Hann [1986]). The unweighted analysis detected a significant $(P<0.001)$ species cooccurrence pattern, but the weighted analysis was nonsignificant $(P=1.000)$ and reduced the standard effect size by 20.64 compared to the unweighted analysis (Appendix B). This large effect of weighting indicated that latitude alone had been responsible for the apparent species co-occurrence pattern in the unweighted analysis.

Despite the more numerous significant results due to weighting, initial predictions were not clearly met. As predicted (Table 1), early/local systems were mixed for the significance of species co-occurrence structure. However, early/regional systems were predicted to be randomly structured, and three of the five systems exhibited significant $(P<0.001)$ structure after weighting, and some weights were substantial in magnitude (Table 2, Appendix B). Also, late/local systems were predicted to consistently exhibit quorum effects, but results were mixed (three of five were significant). Finally, patterns in late/regional systems were predicted to be contingent on the system but uniformly exhibited significant $(P<0.001)$ species co-occurrence patterns that were strongly improved in detectability by weighting.

Area-weighted analyses greatly improved the ability to detect significant checkerboard patterns relative to unweighted analyses: both time and space were significant in ANOVA $(P=0.047$ and 0.006 , respectively), though time-space interaction was not $(P=0.079)$. Related to the nonsignificant interaction term, the distribution of results among temporal/spatial classes was not consistent with quorum effect predictions. In addition, analysis of variance must be considered with caution because assumptions were violated, as for unweighted analyses. Finally, weighted analyses had the greatest effect on regional-scale systems, rather than the temporal/spatial classes that were predicted to be contingent (early/local and late/regional). One more layer of analysis was applied to address apparent scale differences among studies.

\section{Effects of study scale on weighted analyses}

Regional-scale systems included significantly more sites than local-scale systems (ANOVA; $F_{9.9}=24.5 ; P<$ 0.001 ), and systems with more sites also tended to include more species, as evidenced by linear regression $\left(R^{2}=0.405, P=0.003\right)$. The number of study sites bore a strong influence on computed $C$ scores (Fig. 1a) and, most importantly, on area-weighted standard effect sizes (Fig. 1b). Local systems were most closely fit by the regression and so had less residual variance than regional systems (Fig. 1c). Applying weights for study scale consistently reduced area-weighted standard effect sizes, and nine of 13 significant area-weighted results were rendered insignificant by also weighting for study scale (Appendix B). Only four of 17 systems retained significant checkerboard patterns after weighting for both habitat area and study scale, and these four were scattered among three temporal/spatial classes (Table 2, Appendix B). The significant effects of time and space indicated by ANOVA for area-weighted results were negated by further weighting for study scale: neither time $(P=0.411)$, space $(P=0.756)$, nor time-space interaction $(P=0.953)$ were significant by ANOVA (with the same assumption-related cautions as above). 

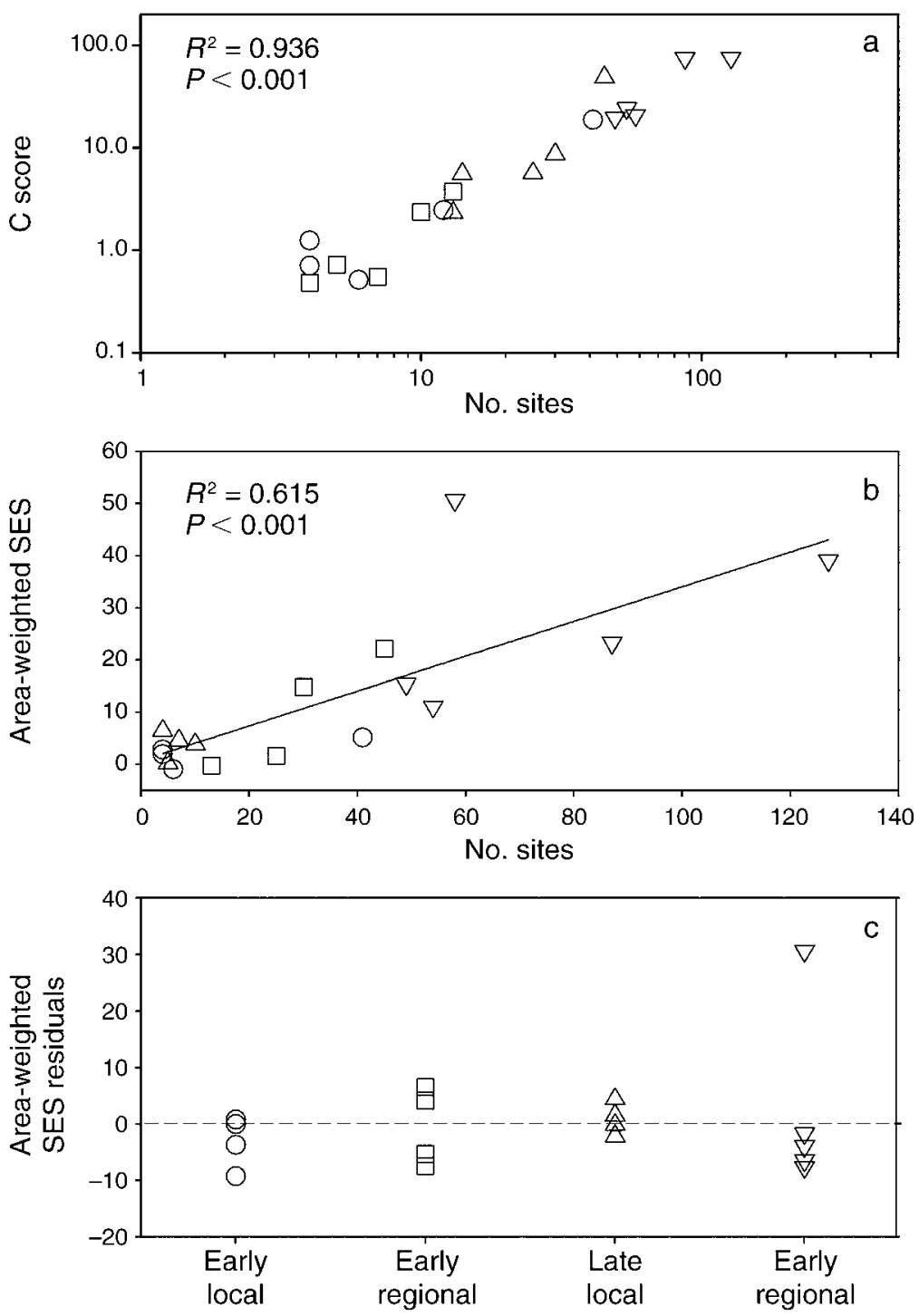

FIG. 1. The effect of study scale (number of study sites) significantly affected (a) computed $C$ scores (note $\log -\log$ scale) and (b) area-weighted standard effect scores (SES). Residuals of the sites vs. area- weighted SES regression (reorganized per temporal/ spatial class in panel c) were used as additional weights in species co-occurrence analyses. See Appendix B for details.

Therefore, quorum conditions could not be generally supported for any temporal/spatial class of studies after checkerboard analyses were weighted for both habitat area and study scale.

Overall, species co-occurrence analyses did not support temporal and spatial quorum effects as predicted for multiple systems: Late/local zooplankton communities were not more likely to have significant species cooccurrence patterns than early/regional communities (Table 2). Habitat area and study scale were important considerations in analyses and were largely responsible for apparent checkerboard patterns in unweighted analyses, as evidenced by the effects of area and study scale on analyses relative to temporal or spatial scales (Table 2).

\section{Discussion}

Species co-occurrence analyses did not consistently indicate a quorum effect in zooplankton community composition, even after accounting for differences in habitat area and scale among studies. I address the three possible arguments of "wrong design, wrong systems, and wrong idea" below, followed by consideration of weighted analyses for other studies.

\section{Temporal/spatial classes}

Some may argue that the a priori assignment of systems to temporal/spatial classes was subjective and potentially inappropriate. However, potential reassignments of systems into other classes would not change the number of significant checkerboard patterns observed. If 
all analyses weighted for area and study scale were rearranged among classes to better suit predictions, the best possible scenario would be that all five systems with significant species co- occurrence structure were dubbed late/local systems (predicted to be in quorum conditions) and nonsignificant systems were early/regional. This scenario would require moving two early/regional plus two late/regional systems into the late/local class and displacing three other systems elsewhere (e.g., early/ regional). If this post hoc rearrangement was performed, late/local systems would include arctic and boreal lakes that span 220-1070 km. Such a rearrangement defies reasonable definitions of both "local" and "late," assuming glaciated regions are biogeographically young for zooplankton, as evidenced by Hebert and Hann (1986) and Stemberger (1995).

The a priori assignment of systems to temporal/spatial classes did not force the evidence that zooplankton communities do not generally exhibit quorum effects. This conclusion is supported by the added caveat that even significant checkerboard patterns can be obtained via stochastic drift processes (Ulrich 2004).

As for any meta-analysis, inclusion of other data sets may have yielded different results, though a wide variety of data sets and temporal/spatial scales were included here. Perhaps more to the point, time and space should be recognized as simple analogs of dispersal rates, which are a function of adaptations, habitat and inter-habitat conditions (including distance), and chance (and therefore time). More knowledge of dispersal kernels (Nathan and Müller-Landau 2000) will enable far better prediction of community assembly outcomes (and thus the conditions for reaching a quorum) than simple time and space categories.

\section{Zooplankton communities}

It may be argued that zooplankton communities are fundamentally different from some other communities and should not be expected to exhibit significant checkerboard structure. In a meta-analysis (Gotelli and McCabe 2002), invertebrates (other than eusocial ants) and other poikilotherms had random species co-occurrence patterns though homeotherms and plants had significant checkerboard structure. If organismal energetics and spatial resource competition underlie significant species co-occurrence patterns (but see Ulrich 2004), then relatively short-lived, invertebrate poikilotherms in dynamic habitats (i.e., zooplankton) should not be expected to consistently derive significant species co-occurrence patterns.

In addition, many zooplankton species undergo an extended dormancy that can involve millions of individuals in a habitat (De Stasio 1989, Hairston et al. 1995, Cáceres and Tessier 2004). Dormancy may delay or avoid local extirpation by a storage effect (Cáceres 1997) and can permit indefinite coexistence in temporally varying habitats (Chase 2005). Quorum effects on colonized species would have to be fierce and consistent for multiple life cycles to affect presence/ absence patterns. Even if local biotic interactions are fierce, interannual variations in that interaction strength plus seasonal succession (i.e., temporal niche partitioning) plus large egg banks operating on diverse temporal cycles may forestall quorum effects from excluding species in subsequent presence/absence data.

\section{The quorum effect}

Finally, one must also question the validity of a quorum effect (i.e., transition from regional to local regulation of community structure) and related ideas such as community assembly time (Mouquet et al. 2003). The quorum metaphor (Jenkins and Buikema 1998) was an attempt to express (and question) the often-presumed priority of local processes as regulators of community structure and function. Analyses here encompassed a wide range of temporal (one to thousands of years) and spatial $(<1 \mathrm{~m}$ to hundreds of kilometers) scales, but did not find consistent evidence of a quorum effect among temporal/spatial classes of zooplankton communities. How can this outcome be reconciled with zooplankton ecology's long history of studies on abiotic and biotic factors as drivers of community structure?

Part of the answer may be that presence/absence data are relatively insensitive to the more subtle effects of local processes (e.g., on birth and death rates). This argument contrasts with recent studies finding significant species co-occurrence patterns (e.g., Gotelli and McCabe 2002, Kobza et al. 2004, Peres-Neto 2004, Badano et al. 2005, Heino and Soininen 2005) but is made more possible by the previous argument regarding zooplankton communities. To put it another way, the sum effects of local processes may only fine-tune the statistics of zooplankton species' presence and may not be strong enough to predictably determine patterns of species' presence and absence among multiple habitats.

Another part of an answer may be that dispersal varies widely among taxa and landscapes (Jenkins and Buikema 1998, Cáceres and Soluk 2002, Bohonak and Jenkins 2003, Louette and De Meester 2005), and so a quorum can be contingent and protracted. Conceptual inclusion of dispersal in niche-based community assembly theory (e.g., Ricklefs 2004, Chase 2005) and progress toward better characterization of zooplankton dispersal (e.g., Michels et al. 2001, Cohen and Shurin 2003, Figuerola et al. 2005) will help address this question, as would coordinated experiments and studies across multiple biogeographic regions and spatial scales.

In terms of the dispersal- vs. niche-based dichotomy of community assembly (Hubbell 2001, Chase and Leibold 2003), meta-analysis yielded results more consistent with the dispersal-based (neutral) model of Hubbell (2001), in that local processes are not indicated to demarcate consistent species co-occurrence patterns through time or across space. However, several nichebased explanations were suggested for these patterns, reflecting recent efforts (e.g., Ricklefs 2004, Chase 2005) 
to build on strengths of each view. The main point is that a single-minded focus on local processes (i.e., interspecific interactions and abiotic conditions) as determinants of species co-occurrence patterns is neither justified nor current.

\section{Lessons for species co-occurrence analyses}

Outcomes of species co-occurrence analyses were strongly affected by the use of weighting factors in analyses, which are less commonly studied than randomization algorithm and null model selection. For example, Gotelli and McCabe (2002) calculated mean habitat area in their meta-analysis, but did not weight analyses by area. This approach may be responsible for the lack of a scale effect on their analyses, whereas the area-weighted analyses presented here were scale-dependent. Of four other recent analyses of species co-occurrence patterns (Kobza et al. 2004, Peres-Neto 2004, Badano et al. 2005, Heino and Soininen 2005), only one (Badano et al. 2005) used weighted analyses despite numerous environmental data collected and analyzed otherwise. Based on the results presented here, weighted analyses should be used far more commonly, especially for habitat area. Additional weighting (e.g., regression residuals for study scale) may not be as widely appropriate for all studies, but the strong study scale effect revealed here on $C$ scores and standard effect scores indicates that sampling efforts should at least be equilibrated among sites before using species co-occurrence analyses, and species co-occurrence meta-analyses should employ weighting for study scale.

\section{ACKNOWLEDGMENTS}

I thank Barbara Taylor, John Havel, and Jurek Kolasa for so kindly sharing their hard-won data and Barbara Taylor, Jon Shurin, and Aaron Ellison for helpful comments on earlier drafts. The idea for this effort was sparked by the "Metacommunities: integrating local and regional processes into community ecology" session at the 2001 ESA annual meeting.

\section{Literature Cited}

Badano, E. I., H. A. Regidor, H. A. Nunez, R. Acosta, and E. Gianoli. 2005. Species richness and structure of ant communities in a dynamic archipelago: effects of island area and age. Journal of Biogeography 32:221-227.

Belyea, L. R., and J. Lancaster. 1999. Assembly rules within a contingent ecology. Oikos 86:402-416.

Bohonak, A. J., and D. G. Jenkins. 2003. Ecological and evolutionary significance of dispersal by freshwater invertebrates. Ecology Letters 6:783-796.

Cáceres, C. E. 1997. Temporal variation, dormancy and coexistence: a field test of the storage effect. Proceedings of the National Academy of Sciences (USA) 94:9171-9175.

Cáceres, C. E., and D. A. Soluk. 2002. Blowing in the wind: a field test of overland dispersal and colonization by aquatic invertebrates. Oecologia 131:402-408.

Cáceres, C. E., and A. J. Tessier. 2004. To sink or swim: variable diapause strategies among Daphnia species. Limnology and Oceanography 49:1333-1340.

Chase, J. M. 2005. Towards a really unified theory for metacommunities. Functional Ecology 19:182-186.

Chase, J. M., and M. A. Leibold. 2003. Ecological niches: linking classical and contemporary approaches. University of Chicago Press, Chicago, Illinois, USA.
Chave, J. 2004. Neutral theory and community ecology. Ecology Letters 7:241-253.

Clements, F. E. 1916. Plant succession, an analysis of the development of vegetation. Carnegie Institution of Washington Publications 242:1-512.

Cohen, G. M., and J. B. Shurin. 2003. Scale-dependence and mechanisms of dispersal in freshwater zooplankton. Oikos 103:603-617.

Connor, E., and D. Simberloff. 1979. The assembly of species communities: Chance or competition? Ecology 60:1132-1140.

Connor, E., and D. Simberloff. 1983. Interspecific competition and species co-occurrence patterns on islands: null models and the evaluation of evidence. Oikos 41:455-465.

Cornell, H. V. 1999. Unsaturation and regional influences on species richness in ecological communities: a review of the evidence. Ecoscience 6:303-315.

Cornell, H. V., and R. H. Karlson. 1996. Species richness of reef-building corals determined by local and regional processes. Journal of Animal Ecology 65:233-241.

Cornell, H. V., and J. H. Lawton. 1992. Species interactions, local and regional processes, and limits to the richness of ecological communities - a theoretical perspective. Journal of Animal Ecology 61:1-12.

Cottenie, K., E. Michels, N. Nuytten, and L. De Meester. 2003. Zooplankton metacommunity structure: regional vs. local processes in highly interconnected ponds. Ecology 84:9911000.

De Stasio, B. T., Jr. 1989. The seed bank of a freshwater crustacean: copepodology for the plant ecologist. Ecology 70: $1377-1389$.

Diamond, J. M. 1975. Assembly of species communities. Pages 342-444 in M. L. Cody and J. M. Diamond, editors. Ecology and evolution of communities. Belknap Press, Cambridge, Massachusetts, USA.

Figuerola, J., A. J. Green, and T. C. Michot. 2005. Invertebrate eggs can fly: evidence of waterfowl-mediated gene flow in aquatic invertebrates. American Naturalist 165:274-280.

Fukami, T. 2004. Assembly history interacts with ecosystem size to influence species diversity. Ecology 85:3234-3242.

Gilpin, M. E., and J. M. Diamond. 1984. Are species cooccurrences on islands nonrandom, and are null hypotheses useful in community ecology? Pages 297-315 in D. R. Strong, Jr., D. Simberloff, L. G. Abele, and A. B. Thistle, editors. Ecological communities: conceptual issues and the evidence. Princeton University Press, Princeton, New Jersey, USA.

Gleason, H. A. 1926. The individualistic concept of the plant association. Bulletin of the Torrey Botanical Club 53:7-26.

Gotelli, N. J. 2000. Null model analysis of species co-occurrence patterns. Ecology 81:2606-2621.

Gotelli, N. J., and G. L. Entsminger. 2002. EcoSim: null models software for ecology. Version 7.0. Acquired Intelligence and Kesey-Bear, Jericho, Vermont, USA.

Gotelli, N. J., and G. L. Entsminger. 2003. Swap algorithms in null model analysis. Ecology 84:532-535.

Gotelli, N. J., and G. R. Graves. 1996. Null models in ecology. Smithsonian Institution Press, Washington, D.C., USA.

Gotelli, N. J., and D. J. McCabe. 2002. Species co-occurrence: a meta-analysis of J. M. Diamond's assembly rules model. Ecology 83:2091-2096.

Gurevitch, J., L. L. Morrow, A. Wallace, and J. S. Walsh. 1992. A meta-analysis of competition in field experiments. American Naturalist 140:539-572.

Hairston, N. G., Jr., R. A. Van Brunt, C. M. Kearns, and D. R. Engstrom. 1995. Age and survivorship of diapausing eggs in a sediment egg bank. Ecology 76:1706-1711.

Havel, J. E., E. M. Eisenbacher, and A. A. Black. 2000. Diversity of crustacean zooplankton in riparian wetlands: colonization and egg banks. Aquatic Ecology 34:63-76.

He, F. L., K. J. Gaston, E. F. Connor, and D. S. Srivastava. 2005. The local-regional relationship: immigration, extinction, and scale. Ecology 86:360-365. 
Hebert, P. D. N., and B. J. Hann. 1986. Patterns in the diversity of arctic zooplankton communities. Canadian Journal of Fisheries and Aquatic Sciences 43:1416-1425.

Heino, J., and J. Soininen. 2005. Assembly rules and community models for unicellular organisms: patterns in diatoms of boreal streams. Freshwater Biology 50:567-577.

Hillebrand, H. 2005. Regressions of local on regional diversity do not reflect the importance of local interactions or saturation of local diversity. Oikos 110:195-198.

Hillebrand, H., and T. Blenckner. 2002. Regional and local impact on species diversity-from pattern to processes. Oecologia 132:479-491.

Hubbell, S. P. 2001. The unified theory of biodiversity and biogeography. Princeton University Press, Princeton, New Jersey, USA.

Hutchinson, G. E. 1967. A treatise on limnology. Volume II. Introduction to lake biology and limnoplankton. John Wiley and Sons, New York, New York, USA.

Jenkins, D. G., and A. L. Buikema, Jr. 1998. Do similar communities develop in similar sites? A test with zooplankton structure and function. Ecological Monographs 68:421433.

Jenkins, D. G., S. Grissom, and K. Miller. 2003. Consequences of prairie wetland drainage for crustacean biodiversity and metapopulations. Conservation Biology 17:158-167.

Kerfoot, W. C., and A. Sih, editors. 1987. Predation: direct and indirect impacts on aquatic communities. University Press of New England, Hanover, New Hampshire, USA.

Kneitel, J. M., and J. M. Chase. 2004. Trade-offs in community ecology: linking spatial scales and species coexistence. Ecology Letters 7:69-80.

Kobza, R. M., J. C. Trexler, W. F. Loftus, and S. A. Perry. 2004. Community structure of fishes inhabiting aquatic refuges in a threatened Karst wetland and its implications for ecosystem management. Biological Conservation 116: $153-165$.

Kolasa, J., J. A. Drake, G. R. Huxel, and C. L. Hewitt. 1996. Hierarchy underlies patterns of variability in species inhabiting natural microcosms. Oikos 77:259-266.

Leibold, M. A., et al. 2004. The metacommunity concept: a framework for multi-scale community ecology. Ecology Letters 7:601-613.

Louette, G., and L. De Meester. 2005. High dispersal capacity of cladoceran zooplankton in newly founded communities. Ecology 86:353-359.

Mahoney, D. L., M. A. Mort, and B. E. Taylor. 1990. Species richness of calanoid copepods, cladocerans and other branchiopods in Carolina bay temporary ponds. American Midland Naturalist 123:244-258.

McCormick, P. V., E. P. Smith, and J. Cairns, Jr. 1991. The relative importance of population versus community processes in microbial primary succession. Hydrobiologia 213: 83-98.

McIntosh, R. P. 1985. The background of ecology: concept and theory. Cambridge University Press, Cambridge, UK.

Michels, E., K. Cottenie, L. Neys, and L. De Meester. 2001. Zooplankton on the move: first results on the quantification of dispersal of zooplankton in a set of interconnected ponds. Hydrobiologia 442:117-126.
Morin, P. J. 1999. Community ecology. Blackwell Science, Malden, Massachusetts, USA.

Mouquet, N., P. Munguia, J. M. Kneitel, and T. E. Miller. 2003. Community assembly time and the relationship between local and regional species richness. Oikos 103:618626.

Nathan, R., and H. C. Müller-Landau. 2000. Spatial patterns of seed dispersal, their determinants and consequences for recruitment. Trends in Ecology and Evolution 15:278-285.

Oksanen, L. 1991. A century of community ecology-How much progress? Trends in Ecology and Evolution 6:294-296.

Paine, R. T. 1977. Controlled manipulations in the marine intertidal zone, and their contributions to ecological theory. Special Publications of the Academy of Natural Sciences, Philadelphia 12:245-270.

Peres-Neto, P. R. 2004. Patterns in the co-occurrence of fish species in streams: the role of site suitability, morphology and phylogeny versus species interactions. Oecologia 140:352360 .

Platt, J. R. 1964. Strong inference. Science 146:347-353.

Price, J. E., and P. J. Morin. 2004. Colonization history determines alternate community states in a food web of intraguild predators. Ecology 85:1017-1028.

Ricklefs, R. E. 1987. Community diversity: relative roles of local and regional processes. Science 235:167-171.

Ricklefs, R. E. 2004. A comprehensive framework for global patterns in biodiversity. Ecology Letters 7:1-15.

Robinson, J., and J. E. Dickerson. 1987. Does invasion sequence affect community structure? Ecology 68:587-595.

Romanuk, T. N., and J. Kolasa. 2002. Environmental variability alters the relationship between richness and variability of community abundances in aquatic rock pool microcosms. Ecoscience 9:55-62.

Rothhaupt, K. O. 1990. Resource competition of herbivorous zooplankton: a review of approaches and perspectives. Archiv für Hydrobiologie 118:1-29.

Shurin, J. B. 2000. Dispersal limitation, invasion resistance, and the structure of pond zooplankton communities. Ecology 81: 3074-3086.

Shurin, J. B., J. E. Havel, M. A. Leibold, and B. Pinel-Alloul. 2000. Local and regional zooplankton species richness: a scale-independent test for saturation. Ecology 81:3062-3073.

Stemberger, R. S. 1995. Pleistocene refuge areas and postglacial dispersal of copepods of the northeastern United States. Canadian Journal of Fisheries and Aquatic Sciences 52:21972210.

Stone, L., and A. Roberts. 1990. The checkerboard score and species distributions. Oecologia 85:74-79.

Swadling, K. M., R. Pienitz, and T. Nogrady. 2000. Zooplankton community composition of lakes in the Yukon and Northwest Territories (Canada): relationship to physical and chemical limnology. Hydrobiologia 431:211-224.

Therriault, T. W., and J. Kolasa. 1999. Physical determinants of richness, diversity, evenness and abundance in natural aquatic microcosms. Hydrobiologia 412:123-130.

Ulrich, W. 2004. Species co-occurrences and neutral models: reassessing J. M. Diamond's assembly rules. Oikos 107:603609.

\section{APPENDIX A}

Analyzed data sets, information about the studied systems, and notes on adjustments to the data sets that were made before analyses (Ecological Archives E087-091-A1).

\section{APPENDIX B}

Species co-occurrence analysis results (Ecological Archives E087-091-A2). 